Article

\title{
Aerobic Training Down-Regulates Pentraxin 3 and Pentraxin 3/Toll-Like Receptor 4 Ratio, Irrespective of Oxidative Stress Response, in Elderly Subjects
}

\author{
Brisamar Estébanez ${ }^{1}{ }^{1}$, Alexandra L. Rodriguez ${ }^{2}$, Nishant P. Visavadiya $^{2}$, Michael Whitehurst $^{2}$, \\ María J. Cuevas ${ }^{1}\left(\mathbb{D}\right.$, Javier González-Gallego ${ }^{1}(\mathbb{D})$ and Chun-Jung Huang ${ }^{2, *(D)}$ \\ 1 Institute of Biomedicine (IBIOMED), University of León, 24007 León, Spain; b.estebanez@unileon.es (B.E.); \\ mj.cuevas@unileon.es (M.J.C.); jgonga@unileon.es (J.G.-G.) \\ 2 Exercise Biochemistry Laboratory, Department of Exercise Science and Health Promotion, Florida Atlantic \\ University, Boca Raton, FL 33431, USA; alexandrarod2014@fau.edu (A.L.R.); nvisavadiya@fau.edu (N.P.V.); \\ whitehur@fau.edu (M.W.) \\ * Correspondence: chuang5@fau.edu; Tel.: +1-561-297-1271
}

Received: 25 December 2019; Accepted: 23 January 2020; Published: 27 January 2020

\begin{abstract}
Reactive oxygen and nitrogen species-mediated cellular aging has been linked to diseases such as atherothrombosis and cancer. Although pentraxin 3 (PTX3) is associated with aging-related diseases via TLR4-dependent anti-inflammatory effects, its relationship with oxidative stress in aging remains to be elucidated. Exercise is proposed as the key intervention for health maintenance in the elderly. This study aimed to examine the association of PTX3 levels with changes in oxidative stress in both plasma and peripheral blood mononuclear cells (PBMCs), following aerobic training in elderly adults. Nine trained and five controls participated in an eight-week aerobic training protocol. Enzyme-linked immunosorbent assay (ELISA) and Western blot analyses were used to determine PTX3, toll-like receptor 4 (TLR4), and levels of oxidative stress biomarkers [3-nitrotyrosine (3NT), 4-hydroxynonenal (4-HNE), glutathione (GSH), protein carbonyl (PC), reactive oxygen/ nitrogen species (ROS/RNS), and trolox equivalent antioxidant capacity (TEAC)] in plasma and/or PBMCs. Results showed a down-regulation of PTX3 expression in PBMCs following aerobic training, along with decreased PTX3/TLR4 ratios. Oxidative stress responses in PBMCs remained unchanged with the exercise protocol. Comparable levels of plasma PTX3 and oxidative stress biomarkers were observed in trained vs. control groups. No correlation was found between PTX3 and any oxidative stress biomarkers following training. These findings demonstrated the down-regulation of PTX3 and PTX3/TLR4 ratio, irrespective of oxidative stress response, in elderly adults following eight weeks of aerobic training.
\end{abstract}

Keywords: aging; endurance training; exercise; pentraxin 3; oxidative stress; toll-like receptor 4 ; inflammation

\section{Introduction}

The normal process of aging has been associated with elevated oxidative stress, as a result of the imbalance between the physiological production of reactive oxygen species (ROS) and antioxidant cell status [1]. ROS are essential to maintain physiological functions, such as gene expression [2], cellular growth [3], and infection defense [4]. Excess production of free radicals can unfavourably modify cellular structural and functional components (e.g., lipids, proteins and DNA) [5], leading to cell senescence as a major risk factor for aging and aging-related diseases (e.g., cardiovascular and neurodegenerative diseases) [6]. One of the possible mechanisms for these ROS-mediated diseases is 
through the activation of intracellular pattern recognition receptors, thereby contributing to a chronic low-grade pro-inflammatory systemic state in aging [5].

Pentraxin 3 (PTX3) is a soluble pattern recognition molecule mainly released from vascular/lymphatic endothelial cells and immunes cells (neutrophils and monocytes) [7] following immune stimulation (e.g., tumor necrosis factor- $\alpha$ and toll-like receptor (TLR) agonists) [8]. PTX3 has been shown to utilize its counter-regulatory function in promoting an anti-inflammatory response [7] via the inhibition of TLR4 [9]. In particular, research has recently attempted to understand the cross-regulatory role between PTX3 and oxidative stress. For example, a recent study demonstrated an increased level of PTX3 following stimulation of oxidative stress [4-hydroxynonenal (4-HNE); an end product of lipid peroxidation] in human retinal pigmented epithelium cells [10]. Furthermore, Balci et al. [11] observed a positive correlation of PTX3 with total oxidative stress, whereas PTX3 was negatively correlated with total antioxidant capacity. However, although increased PTX3 plasma levels have been associated with aging-related diseases, including chronic kidney disease [12], the relationship between PTX3 and oxidative stress in aging remains to be elucidated.

Regular physical activity, such as aerobic training, has been proposed as a successful intervention to delay the physiological processes of aging, ameliorating the aging-related decline in the immune response [13], arterial stiffening [14], sarcopenia [15] or cognitive function [16], and other associated diseases, such as cardiovascular diseases [17], Alzheimer's disease, and Parkinson's disease [18]. Generally, during acute physical exercise blood flow is redistributed from inactive muscle to active skeletal muscle and the brain, although eventually all organs obtain the exercise-promoted beneficial effects with training [19]. However, even though some molecular processes, such as TLRs and NLRP3 inflammasome pathways activation, autophagy, apoptosis, mitochondrial function, or unfolded protein response to endoplasmic reticulum stress have been evaluated in response to exercise in aging [20-24], the potential effect of training adaptation remains to be elucidated. Specifically, the literature has previously demonstrated that aerobic training provided health benefits including the down-regulation of oxidative stress and/or increasing antioxidant activity in older adults [25]. These findings have been further supported by Park and Kwak [26], demonstrating lower oxidative stress responses [protein carbonyl (PC) and malondialdehyde (MDA)], but higher levels of total antioxidant capacity (TEAC) in aerobically trained vs. untrained individuals following acute intense exercise. Of particular note, Bouzid et al. [27] found that both young-aged sedentary and old-aged active adults elicited comparable levels of acute exercise-induced MDA and antioxidant activity, suggesting an attenuation of aging-related ROS or improvement of antioxidant defense with training.

Our group has recently investigated the effect of acute aerobic exercise on plasma PTX3 response and showed an elevation in both healthy normal-weight $[7,28,29]$ and obese individuals [30-32], regardless of fitness status. In addition, the plasma levels of PTX3 were upregulated following eight weeks of aerobic training in middle-aged and older adults [33,34]. While PTX3 augments the anti-inflammatory response [7] via the inhibition of TLR4 [9], McFarlin et al. [35] found a lower expression of lipopolysaccharide stimulated TLR4 on monocytes when comparing active to inactive elderly individuals. Furthermore, this down-regulation of TLR4 was also observed in the elderly following 12 weeks of concurrent aerobic and strength training [36] and 8 weeks of either resistance training [13] or whole-body vibration program [37]. More importantly, Farinha et al. [38] examined the relationship between oxidative stress and inflammation in untrained middle-aged women with metabolic syndrome, in response to a 12-week aerobic training, and showed decreased levels of oxidative stress biomarkers (advanced oxidation protein products and thiobarbituric acid-reactive substances) as well as pro-inflammatory cytokines (e.g., interleukin-6), while the levels of the anti-inflammatory cytokine IL-10 increased.

Therefore, this study was to examine whether or not the level of PTX3 on TLR4-dependennt inflammation would be associated with changes in oxidative stress in both plasma and peripheral blood mononuclear cells (PBMCs) following aerobic training in the elderly. 


\section{Materials and Methods}

\subsection{Subjects}

Fourteen healthy elderly subjects (9 trained ( 2 males and 7 females; $68.68 \pm 1.24$ years) and 5 controls ( 2 males and 3 females, $70.78 \pm 1.51$ years) ) volunteered to participate in the present study (see participant characteristics in Table 1). Prior to data collection, all subjects completed the informed consent form, a medical history questionnaire, a physical activity readiness questionnaire (PAR-Q), a risk factor quiz, and an electrocardiogram test. All experimental procedures followed the principles of the Declaration of Helsinki and were approved by the local ethics committee (\#25-2013). Of note, the subject cohort in this study was a subset of our previous publication [20].

Table 1. Participant anthropometric characteristics.

\begin{tabular}{cccc}
\hline Variable & CG $(\mathbf{n}=\mathbf{5})$ & TG $(\mathbf{n}=\mathbf{9 )}$ & $p$ Value \\
\hline Age $($ years $)$ & $70.79 \pm 1.66$ & $68.67 \pm 1.25$ & 0.330 \\
Height $(\mathrm{cm})$ & $160.60 \pm 0.03$ & $159.50 \pm 0.034$ & 0.825 \\
Weight $(\mathrm{kg})$ & $67.00 \pm 4.36$ & $65.63 \pm 3.87$ & 0.828 \\
BMI $\left(\mathrm{kg} / \mathrm{m}^{2}\right)$ & $25.87 \pm 1.07$ & $25.71 \pm 0.90$ & 0.914 \\
$\mathrm{VO}_{2 \text { peak }}(\mathrm{mL} / \mathrm{kg} / \mathrm{min})$ & $30.37 \pm 1.44$ & $31.02 \pm 1.58$ & 0.789 \\
\hline CG $=$ Control Group; TG $=$ Trained Group; $\mathrm{VO}_{2 \text { peak }}=$ Peak Oxygen Uptake.
\end{tabular}

Subjects that possessed any known inflammatory diseases/conditions (e.g., cardiovascular disease, diabetes) or were taking a medical drug, antioxidant supplementation and hormonal treatment were excluded to limit the potential effects of outcome variables. Subjects were also excluded if they participated in any aerobic exercise training within the past year. Finally, during the study period, subjects were instructed to maintain their regular physical activity and diets.

\subsection{Experimental Protocol}

Aerobic exercise training comprised 16 sessions of cycling protocols over a period of 8 weeks ( 2 sessions per week) on a stationary ergometer (Tunturi Bike F35, Almere, the Netherlands), with at least $48 \mathrm{~h}$ between sessions. Each session consisted of 25-30 min of total exercise, including a $5 \mathrm{~min}$ warm-up, followed by 15-20 min at an intensity of 70-75\% of maximum heart rate, along with a 5 min of active recovery. Short periods of high intensity activity ( $1 \mathrm{~min}$; $90-95 \%$ of maximum heart rate) were progressively introduced across sessions [20].

\subsection{Blood Sampling}

Venous blood samples $(30 \mathrm{~mL})$ were collected by a trained phlebotomist from the brachiocephalic vein prior to and following 8 weeks of training using the EDTA anticoagulant Vacutainer ${ }^{\mathrm{TM}}$ systems (BD, Franklin Lakes, NJ, USA). To avoid circadian effects, all blood samples were collected between 8:00 a.m. and 9:00 a.m. Subjects were asked to fast overnight for $12 \mathrm{~h}$ and abstained from alcohol, caffeine intake, and intense physical activity for at least 5-6 days prior to each blood collection. Finally, plasma and PBMCs were collected from whole blood and stored at $-80^{\circ} \mathrm{C}$ for further analyses.

\subsection{Plasma Assays}

The plasma pentraxin 3 (cat\# ab214570, Abcam, Cambridge, UK), reduced glutathione (GSH, cat\# E-BC-K030, Elabscience, Texas, USA) and total antioxidant capacity (TEAC) (ABTS assay, cat\# AOX-1, Zenbio, Research Triangle Park, NC, USA) were measured through commercially available kits with the Epoch ${ }^{\mathrm{TM}}$ microplate spectrophotometer (BioTek Instruments, Winooski, VT, USA). The plasma total reactive oxygen species and reactive nitrogen species (ROS/RNS) were measured using the $2^{\prime}, 7^{\prime}$-Dichlorofluorescin diacetate fluorescent probe (DCFH2-DA, cat\# D6883, Millipore Sigma, St. Louis, MO, USA). Briefly, $10 \mu \mathrm{L}$ plasma was incubated with $90 \mu \mathrm{L}$ of DCFH2-DA probe (final concentration $10 \mu \mathrm{M}$ ) in PBS, pH 7.4 at room temperature for $10 \mathrm{~min}$ in the dark. After the incubation 
period, the oxidized DCF fluorescence was measured using ( $485 \mathrm{~nm}$ excitation/520 $\mathrm{nm}$ emission filters) a BioTek Synergy HTX spectrofluorometer (Winooski, VT, USA).

\subsection{Isolation of $P B M C S$}

To isolate PBMCs, blood was layered over a Ficoll separation solution ( $\varrho=1.077 \mathrm{~g} / \mathrm{mL}$; Sigma-Aldrich, St. Louis, MO, USA) and centrifuged at $400 \times \mathrm{g}$ for $30 \mathrm{~min}$ at room temperature. PBMC layer was washed in saline buffer phosphate, $\mathrm{pH}$ 7.4, and PBMCs were lysated using a buffer $\mathrm{pH}$ 7.4, constituted by $0.25 \mathrm{mM}$ sucrose, $1 \mathrm{mM}$ EDTA, $10 \mathrm{mM}$ Tris and a standard protease and phosphatase inhibitor cocktail (Sigma-Aldrich, St. Louis, MO, USA). A Bradford assay was used to determinate protein quantification.

\subsection{Western Blot Analysis}

A total of $40 \mu \mathrm{g}$ of PBMC proteins were separated by molecular weight using a SDS-PAGE, 4\%-20\% Criterion ${ }^{\mathrm{TM}}$ TGX ${ }^{\mathrm{TM}}$ Precast gels (cat\# 5671095, Bio-Rad, Hercules, CA, USA). The gel proteins were transblotted onto polyvinylidene difluoride (PVDF) membranes and incubated in $5 \%$ nonfat dry milk for an hour at room temperature. Further, blots were incubated at $4{ }^{\circ} \mathrm{C}$ overnight with a primary antibody against PTX3 (1:5000, cat\# ab125007, Abcam, Cambridge, UK), 4-HNE (1:1000, cat\# ab46545, Abcam, Cambridge, UK), 3-nitrotyrosine (3NT) (1:1000, cat\# 9691, Cell Signaling Technology Inc), TLR4 (1:500, cat\# 293072, Santa Cruz Biotechnology) or GAPDH (1:5000, cat\# 97166, Cell Signaling Technology Inc) in 5\% nonfat dry milk. The protein carbonyls (PC) detection was performed according to manufacturer's instruction using an OxyBlot kit (cat\# S7150; Millipore Inc). For secondary antibodies, peroxidase-conjugated horse anti-mouse IgG (cat\# 7076) and goat anti-rabbit IgG (cat\# 7074) from Cell Signaling Technology Inc were used. The immunoreactive protein reaction was exposed in ChemiDocTM XRS+ imaging system (Bio-rad) using a SuperSignal ${ }^{\mathrm{TM}}$ West Pico PLUS Chemiluminescent substrate solution (cat\# PI34580, Thermo Fisher), and band density analysed by the ImageJ software (NIH, Bethesda, MD, USA).

\subsection{Statistical Analysis}

All statistical analysis was performed using SPSS version 25.0 (SPSS Inc., Chicago, IL, USA). Normality of the data was confirmed with a Shapiro-Wilk test. Baseline differences between both trained and control groups were conducted using independent $t$-tests. A two group (trained vs. control) $\times$ two time points (pre vs. post) repeated measures analyses of variance (ANOVA) was utilized to examine the effect of 8 weeks of aerobic training on the plasma levels of PTX3, GSH, TEAC, and ROS/RNS and the expression of PTX3, TLR4, 3NT, 4-HNE, and PC in PBMCs. The Greenhouse-Geisser correction of degrees of freedom was used when sphericity assumptions were violated, and significant effects were further analyzed with Bonferroni post hoc comparisons. Furthermore, Pearson's product-moment correlations were used to examine the relationships in outcome variables between both levels of plasma and PBMCs. Significant differences were defined as $p<0.05$. Data are presented as mean \pm standard error of means (SEM).

\section{Results}

\subsection{Measurements of Inflammatory and Oxidative Biomarkers at Baseline}

Our analyses confirmed no difference in the baseline level of plasma PTX3 between elderly trained and control groups $(\mathrm{t}[12]=0.021, p=0.984)$. Likewise, the trained group did not show any differences in plasma markers of oxidative stress at baseline than controls: GSH ( $\mathrm{t}[12]=-0.152, p=0.881)$, TEAC $(\mathrm{t}[12]=0.266, p=0.795)$ and ROS/RNS ( $\mathrm{t}[12]=-0.210, p=0.837)$. To further verify inflammatory levels in PBMCs at baseline, our results demonstrated comparable expression of PTX3 (t [12] $=1.318$, $p=0.212)$, TLR4 ( $\mathrm{t}[12]=0.847, p=0.414)$ and PTX3/TLR4 ratio $(\mathrm{t}[12]=0.163, p=0.873)$ in the elderly trained group compared to controls. Finally, no difference was found in the baseline levels of 
oxidative stress biomarkers: $\mathrm{PC}\left(\mathrm{t}_{[4.755]}=2.559, p=0.053\right), 4-\mathrm{HNE}(\mathrm{t}[12]=-0.697, p=0.499)$, and 3NT $(\mathrm{t}[12]=-0.967, p=0.353)$ in PBMCs between both groups.

\subsection{Effects of Aerobic Training on Inflammatory and Oxidative Stress Responses}

As illustrated in Figure 1, aerobic exercise training did not show any significant changes in both PTX3 $\left(\mathrm{F}_{[1,14]}=0.391, p=0.543\right)$ and oxidative stress biomarkers: $\mathrm{GSH}\left(\mathrm{F}_{[1,14]}=0.108, p=0.748\right)$, $\operatorname{TEAC}\left(\mathrm{F}_{[1,14]}=0.426, p=0.526\right)$, and ROS/RNS $\left(\mathrm{F}_{[1,14]}=0.004, p=0.949\right)$ in plasma between elderly trained vs. control subjects.

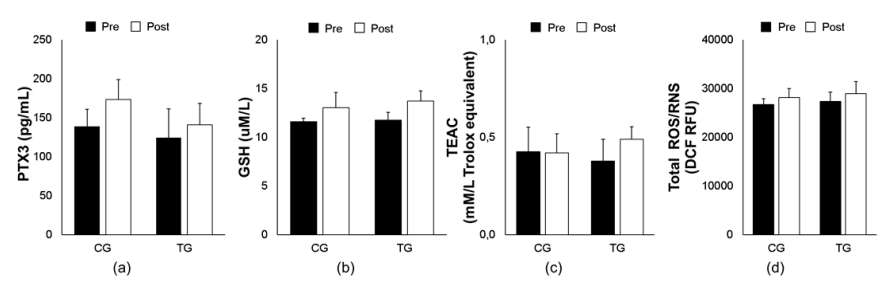

Figure 1. Effects of an 8-week aerobic training on plasma PTX3 (a) and oxidative biomarkers (GSH (b), TEAC (c), and total ROS/RNS (d)) in both trained (TG) and control (CG) groups. Data are means \pm SEM. GSH, reduced glutathione; PTX3, pentraxin 3; ROS/RNS, reactive oxygen/ nitrogen species; TEAC, trolox equivalent antioxidant capacity.
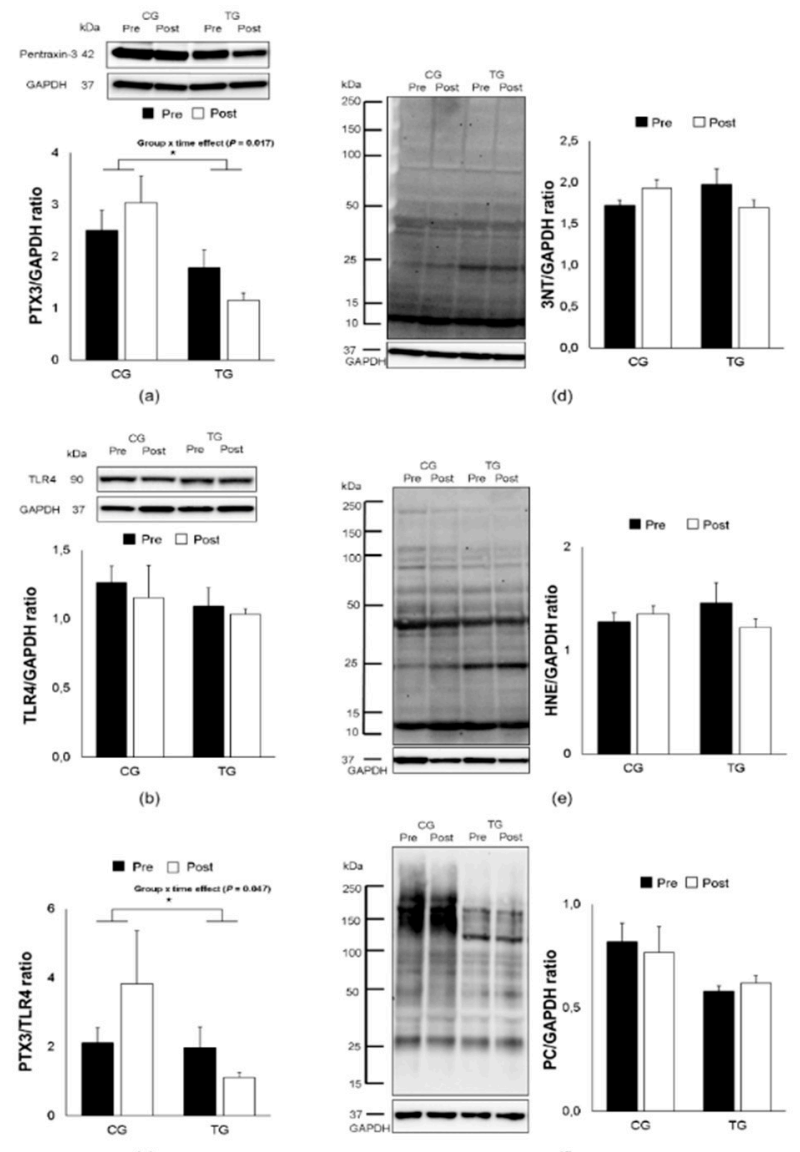

Figure 2. Effects of an 8-week aerobic training on PTX3, TLR4, PTX3/TLR4 ratio, 3NT, 4-HNE and PC expression. Representative Western blots and densitometric quantification of PTX3 (a), TLR4 (b), PTX3/TLR4 ratio (c) 3NT (d), 4-HNE (e), and PC (f) in PBMCs from elderly trained (TG) and control $(\mathrm{CG})$ groups. Data are means \pm SEM. The * indicates a significant $(p<0.05)$ group $\times$ time effect. 3NT, 3-nitrotyrosine; 4-HNE, 4-hydroxynonenal; PC, protein carbonyl; TLR4, toll-like receptor 4. 
However, the training protocol demonstrated a significant group-by-time effect with a down-regulative expression of PTX3 in PBMCs in the elderly trained subjects $\left(\mathrm{F}_{[1,14]}=7.735\right.$, $p=0.017$, Figure 2a). Furthermore, the PTX3/TLR4 ratio was significantly decreased following aerobic training $\left(\mathrm{F}_{[1,14]}=4.917, p=0.047\right.$, Figure $\left.2 \mathrm{c}\right)$, whereas no change was found in the TLR4 expression $\left(\mathrm{F}_{[1,14]}=0.045, p=0.836\right.$, Figure $\left.2 \mathrm{~b}\right)$. The tendency of oxidative stress responses in PBMCs for 3NT, 4-HNE, and PC remained unchanged as shown in plasma levels $\left(\mathrm{F}_{[1,14]}=2.739, p=0.124\right.$, Figure 2d; $\mathrm{F}_{[1,14]}=1.534, p=0.239$, Figure $2 \mathrm{e} ; \mathrm{F}_{[1,14]}=1.347, p=0.268$, Figure 2f; respectively). Finally, no correlation was observed between PTX3 and any oxidative stress biomarkers following aerobic training.

\section{Discussion}

This study was to investigate the effects of an eight-week aerobic training on the inflammatory and oxidative stress responses in both plasma and PBMCs of elderly subjects. Our results did not demonstrate any changes in plasma levels of PTX3 and oxidative stress biomarkers in elderly trained group compared to controls following the training protocol. However, aerobic training mediated a down-regulation of PTX3 expression in PBMCs, along with decreased ratio of PTX3/TLR4, whereas the expression of oxidative stress biomarkers in PBMCs remained unchanged as shown in plasma levels. These findings demonstrated the down-regulative expression of PTX3 and PTX3/TLR4 ratio in PBMCs of elderly subjects, irrespective of changes in oxidative stress following eight weeks of aerobic training.

The literature has shown elevated levels of oxidative stress in elderly adults [39]. Although acute bouts of exercise can elicit the release of oxidative stress biomarkers in plasma [40], aerobic exercise training has been proposed to attenuate this oxidative status [41]. For example, a three-month endurance cycling training protocol reduced 4-HNE and PC but increased antioxidant manganese superoxide dismutase (MnSOD) levels in skeletal muscle from obese subjects [42]. Moreover, a decrease in oxidative stress biomarker, F2-isoprostane, in older women was found following a 12-month aerobic training [43]. However, the present study did not observe any changes in oxidative stress biomarkers following aerobic training. These findings are in concordance with existing literature, indicative of no effects of an eight-week training protocol on oxidative stress response [44]. One of possible explanations for this insignificant change in oxidative status may be due to insufficient training duration and/or intensity, particularly in the elderly. Specifically, Done and Traustadóttir [45] also examined the effect of an eight-week aerobic training on oxidative stress response in older adults and showed a similar result with no change in F2-isoprostanes. However, the study by Done and Traustadóttir [45] found that eight weeks of aerobic training is sufficient in improving resistance to oxidative stress as measured by F2-isoprostane response to a forearm ischemia/reperfusion (I/R), suggesting that this increased resistance to oxidative stress may be associated with improved cardiorespiratory fitness $\left(\mathrm{VO}_{2 \mathrm{max}}\right.$ following training. Importantly, the training protocol in our elderly subjects also demonstrated increased peak oxygen uptake [20]. Thus, future investigation should include the measure of resistance to oxidative stress in conjunction with extended training duration to gain a further understating of ROS-mediated cellular aging and associated diseases with aerobic training.

No studies have examined the effect of aerobic training on plasma level of PTX3 response, along with its expression in PBMCs of elderly adults. Although there is limited literature in comparing PTX3 levels between young and elderly adults, an elevation in PTX3 plasma levels has been associated with aging-related diseases [12]. Specifically, Krzanowski et al. [46] found that baseline levels of PTX3 could predict cardiovascular mortality in patients with advanced chronic kidney disease. This finding is supported by Lee et al. [12], demonstrating a positive relationship between plasma PTX3 levels and risk of chronic kidney disease in elderly adults, while the biological and molecular mechanisms still remain to be elucidated. Importantly, exercise has been established as an important modulator of the PTX3 expression in response to both acute and chronic exercise training. For example, an increase in plasma PTX3 levels after a single bout of aerobic exercise has been shown in both healthy normal-weight $[7,28,29]$ and obese individuals [30-32]. Although the training protocol in the present study did not observe any change in the level of plasma PTX3, research has previously demonstrated 
a significant elevation in plasma level of PTX3 in in middle-aged and older adults following an eight-week aerobic training with an intensity of 60-70\% peak oxygen uptake for 30-45 min, three days per week $[33,34]$. Thus, the insignificant result of plasma PTX3 in this study was most likely due to a lower training intensity (duration: $25-30 \mathrm{~min}$ vs. 30-45 min and frequency: 2 vs. 3 sessions/week). However, to our knowledge, this study was the first to demonstrate a down-regulation of PTX3 expression in PBMCs, along with decreased ratio of PTX3/TLR4 following aerobic training, although no relationship was found between PTX3 and oxidative stress status.

It is important to note that this reduced ratio of PTX3/TLR4 is mainly attributed to deceased PTX3 expression, but not TLR4 modifications. This absence of change in TLR4 may be due to the type of training performed, since our group previously found a decrease in TLR4 expression following eight weeks of both resistance training [13] and whole-body vibration program [37]. While acute eccentric exercise was previously found to increase the expression of TLR4 [47], we also demonstrated that six weeks of eccentric training was sufficient to down-regulate TLR4 expression in PBMCs from young adults [48]. However, the findings of this study may be partially supported by the reduced endotoxin-stimulated PTX3 in PBMCs immediately following acute aerobic exercise [32], which may suggest that the mechanisms associated with PTX3-mediated anti-inflammatory signaling are enhanced in the elderly with aerobic training.

\section{Conclusions}

This study demonstrated that aerobic training down-regulates the expression of PTX3 and PTX3/TLR4 ratio in PBMCs of elderly subjects, regardless of variations in oxidative stress status. These results provide an insight into the possible mechanisms of PTX3-mediated anti-inflammatory signaling in the elderly with aerobic training. Future studies with a larger sample size and extended training protocol are warranted to further elucidate the protective role of PTX3 on cellular aging and associated diseases.

Author Contributions: Conceptualization, B.E., N.P.V., M.J.C., J.G.-G., and C.-J.H.; formal analysis, B.E., N.P.V., and C.-J.H.; funding acquisition, M.W. and C.-J.H.; investigation, B.E., A.L.R., N.P.V., and C.-J.H.; methodology, B.E., N.P.V., M.J.C., J.G.-G., and C.-J.H.; project administration, B.E. and C.-J.H.; resources, M.W., M.J.C., J.G.-G., and C.-J.H.; supervision, M.J.C., J.G.-G., and C.-J.H.; validation, B.E. and C.-J.H.; visualization, B.E., N.P.V., and C.-J.H.; writing—original draft, B.E. and C.-J.H.; writing—review and editing, B.E., A.L.R., N.P.V., M.W., M.J.C., J.G.-G., and C.-J.H. All authors have read and agree to the published version of the manuscript.

Funding: Funding for this project was provided by the Department of Exercise Science and Health Promotion at Florida Atlantic University. B. Estébanez was supported by a fellowship from the Ministry of Education of Spain (FPU fellowship, reference FPU15/05051).

Conflicts of Interest: The authors declare no conflicts of interest.

\section{References}

1. Chen, F.; Liu, Y.; Wong, N.-K.; Xiao, J.; So, K.-F. Oxidative stress in stem cell aging. Cell Transplant. 2017, 26, 1483-1495. [CrossRef]

2. Han, E.-S.; Muller, F.L.; Pérez, V.I.; Qi, W.; Liang, H.; Xi, L.; Fu, C.; Doyle, E.; Hickey, M.; Cornell, J.; et al. The in vivo gene expression signature of oxidative stress. Physiol. Genom. 2008, 34, 112-126. [CrossRef]

3. Lelkes, P.I.; Hahn, K.A.; Karmiol, S.; Schmidt, D.H. Hypoxia/reoxygenation enhances tube formation of cultured human microvascular endothelial cells: The role of reactive oxygen species. In Angiogenesis; Springer Science and Business Media LLC: Berlin/Heidelberg, Germany, 1998; pp. 321-336.

4. Ivanov, A.V.; Valuev-Elliston, V.T.; Ivanova, O.N.; Kochetkov, S.N.; Starodubova, E.S.; Bartosch, B.; Isaguliants, M.G. Oxidative stress during HIV infection: Mechanisms and consequences. Oxidative Med. Cell. Longev. 2016, 2016, 1-18. [CrossRef] [PubMed]

5. Bullone, M.; Lavoie, J.-P. The contribution of oxidative stress and inflamm-aging in human and equine asthma. Int. J. Mol. Sci. 2017, 18, 2612. [CrossRef] [PubMed]

6. Cai, Z.; Zhang, J.; Li, H. Selenium, aging and aging-related diseases. Aging Clin. Exp. Res. 2019, 31, 1035-1047. [CrossRef] [PubMed] 
7. Slusher, A.L.; Whitehurst, M.; Maharaj, A.; Dodge, K.M.; Fico, B.G.; Mock, J.T.; Huang, C.-J. Plasma pentraxin 3 and glucose kinetics following acute high-intensity interval exercise versus continuous moderate-intensity exercise in healthy men. Appl. Physiol. Nutr. Metab. 2018, 43, 1233-1238. [CrossRef]

8. Alles, V.V.; Bottazzi, B.; Peri, G.; Golay, J.; Introna, M.; Mantovani, A. Inducible expression of PTX3, a new member of the pentraxin family, in human mononuclear phagocytes. Blood 1994, 84, 3483-3493. [CrossRef]

9. Bozza, S.; Campo, S.; Arseni, B.; Inforzato, A.; Ragnar, L.; Bottazzi, B.; Mantovani, A.; Moretti, S.; Oikonomous, V.; De Santis, R.; et al. PTX3 binds MD-2 and promotes TRIF-dependent immune protection in aspergillosis. J. Immunol. 2014, 193, 2340-2348. [CrossRef]

10. Wang, L.; Cano, M.; Datta, S.; Wei, H.; Ebrahimi, K.B.; Gorashi, Y.; Garlanda, C.; Handa, J.T. Pentraxin 3 recruits complement factor $\mathrm{H}$ to protect against oxidative stress-induced complement and inflammasome overactivation. J. Pathol. 2016, 240, 495-506. [CrossRef]

11. Balci, Y.I.; Nuray, E.; Polat, A.; Enli, Y.; Ozgurler, F.; Akin, M.; Balc1, Y.I.; Akın, M. Pentraxin-3 levels in beta thalassemia major and minor patients, and its relationship with antioxidant capacity and total oxidant Stress. J. Pediatr. Hematol. 2016, 38, 1-16.

12. Lee, R.; Shin, M.-H.; Kim, H.-N.; Lee, Y.-H.; Choi, S.-W.; Ahn, H.-R.; Kweon, S.-S. Relationship between plasma pentraxin 3 level and risk of chronic kidney disease in the Korean elderly: The Dong-gu study. Int. Urol. Nephrol. 2017, 49, 2027-2033. [CrossRef]

13. Rodriguez-Miguelez, P.; Fernandez-Gonzalo, R.; Almar, M.; Mejías, Y.; Rivas, A.; De Paz, J.A.; Cuevas, M.J.; González-Gallego, J. Role of Toll-like receptor 2 and 4 signaling pathways on the inflammatory response to resistance training in elderly subjects. AGE 2014, 36, 9734. [CrossRef]

14. Fujie, S.; Hasegawa, N.; Sanada, K.; Hamaoka, T.; Maeda, S.; Padilla, J.; Martinez-Lemus, L.A.; Iemitsu, M. Increased serum salusin- $\alpha$ by aerobic exercise training correlates with improvements in arterial stiffness in middle-aged and older adults. Aging 2020. [CrossRef] [PubMed]

15. Naseeb, M.A.; Volpe, S.L. Protein and exercise in the prevention of sarcopenia and aging. Nutr. Res. 2017, 40, 1-20. [CrossRef] [PubMed]

16. Northey, J.M.; Cherbuin, N.; Pumpa, K.L.; Smee, D.J.; Rattray, B. Exercise interventions for cognitive function in adults older than 50: A systematic review with meta-analysis. Br. J. Sports Med. 2018, 52, 154-160. [CrossRef] [PubMed]

17. Ross, M.D. Aging-How Lifestyle changes could prove to be an effective medicine for the aging cardiovascular system. Curr. Cardiol. Rev. 2018, 14, 225-226. [CrossRef]

18. Paillard, T.; Rolland, Y.; Barreto, P.D.S. Protective effects of physical exercise in Alzheimer's disease and Parkinson's disease: A narrative review. J. Clin. Neurol. 2015, 11, 212-219. [CrossRef]

19. Radak, Z.; Torma, F.; Berkes, I.; Goto, S.; Mimura, T.; Posa, A.; Balogh, L.; Boldogh, I.; Suzuki, K.; Higuchi, M.; et al. Exercise effects on physiological function during aging. Free. Radic. Boil. Med. 2019, 132, 33-41. [CrossRef]

20. Mejías-Peña, Y.; Rodriguez-Miguelez, P.; Fernandez-Gonzalo, R.; Martínez-Flórez, S.; Almar, M.; De Paz, J.A.; Cuevas, M.J.; González-Gallego, J. Effects of aerobic training on markers of autophagy in the elderly. AGE 2016, 38, 33. [CrossRef]

21. Mejías-Peña, Y.; Estébanez, B.; Rodriguez-Miguelez, P.; Fernandez-Gonzalo, R.; Almar, M.; De Paz, J.A.; González-Gallego, J.; Cuevas, M.J. Impact of resistance training on the autophagy-inflammation-apoptosis crosstalk in elderly subjects. Aging 2017, 9, 408-418. [CrossRef]

22. Estébanez, B.; Moreira, O.C.; Almar, M.; De Paz, J.A.; Gonzalez-Gallego, J.; Cuevas, M.J. Effects of a resistance-training programme on endoplasmic reticulum unfolded protein response and mitochondrial functions in PBMCs from elderly subjects. Eur. J. Sport Sci. 2019, 19, 931-940. [CrossRef] [PubMed]

23. Moreira, O.C.; Estébanez, B.; Martínez-Florez, S.; De Paz, J.A.; Cuevas, M.J.; González-Gallego, J. Mitochondrial function and mitophagy in the elderly: Effects of exercise. Oxidative Med. Cell. Longev. 2017, 2017, 1-13. [CrossRef] [PubMed]

24. Estébanez, B.; De Paz, J.A.; Cuevas, M.J.; González-Gallego, J. Endoplasmic reticulum unfolded protein response, aging and exercise: An update. Front. Physiol. 2018, 9, 1744. [CrossRef] [PubMed]

25. Fatouros, I.G.; Jamurtas, A.Z.; Villiotou, V.; Pouliopoulou, S.; Fotinakis, P.; Taxildaris, K.; Deliconstantinos, G. Oxidative stress responses in older men during endurance training and detraining. Med. Sci. Sports Exerc. 2004, 36, 2065-2072. [CrossRef] 
26. Park, S.-Y.; Kwak, Y.-S. Impact of aerobic and anaerobic exercise training on oxidative stress and antioxidant defense in athletes. J. Exerc. Rehabilitation 2016, 12, 113-118. [CrossRef]

27. Bouzid, M.A.; Filaire, E.; Matran, R.; Robin, S.; Fabre, C. Lifelong voluntary exercise modulates age-related changes in oxidative stress. Int. J. Sports Med. 2018, 39, 21-28. [CrossRef]

28. Huang, C.-J.; Webb, H.E.; Beasley, K.N.; McAlpine, D.A.; Tangsilsat, S.E.; Acevedo, E.O. Cardiorespiratory fitness does not alter plasma pentraxin 3 and cortisol reactivity to acute psychological stress and exercise. Appl. Physiol. Nutr. Metab. 2014, 39, 375-380. [CrossRef]

29. Slusher, A.L.; Zúniga, T.M.; Acevedo, E.O. Aerobic fitness alters the capacity of mononuclear cells to produce pentraxin 3 following maximal exercise. Graefe's Arch. Clin. Exp. Ophthalmol. 2018, 118, 1515-1526. [CrossRef]

30. Slusher, A.L.; Mock, J.T.; Whitehurst, M.; Maharaj, A.; Huang, C.-J. The impact of obesity on pentraxin 3 and inflammatory milieu to acute aerobic exercise. Metab. 2015, 64, 323-329. [CrossRef]

31. Slusher, A.L.; Huang, C.-J. Association of pentraxin 3 with insulin resistance and glucose response following maximal aerobic exercise in obese and normal-mass individuals. Can. J. Physiol. Pharmacol. 2016, 94, 734-738. [CrossRef]

32. Slusher, A.L.; Shibata, Y.; Whitehurst, M.; Maharaj, A.; Quiles, J.M.; Huang, C.-J. Exercise reduced pentraxin 3 levels produced by endotoxin-stimulated human peripheral blood mononuclear cells in obese individuals. Exp. Boil. Med. 2017, 242, 1279-1286. [CrossRef] [PubMed]

33. Miyaki, A.; Maeda, S.; Choi, Y.; Akazawa, N.; Tanabe, Y.; Ajisaka, R. Habitual aerobic exercise increases plasma pentraxin 3 levels in middle-aged and elderly women. Appl. Physiol. Nutr. Metab. 2012, 37, 907-911. [CrossRef] [PubMed]

34. Zempo-Miyaki, A.; Fujie, S.; Sato, K.; Hasegawa, N.; Sanada, K.; Maeda, S.; Hamaoka, T.; Iemitsu, M. Elevated pentraxin 3 level at the early stage of exercise training is associated with reduction of arterial stiffness in middle-aged and older adults. J. Hum. Hypertens. 2016, 30, 521-526. [CrossRef] [PubMed]

35. McFarlin, B.K.; Flynn, M.G.; Campbell, W.W.; Craig, B.A.; Robinson, J.P.; Stewart, L.K.; Timmerman, K.L.; Coen, P.M. Physical activity status, but not age, influences inflammatory biomarkers and toll-like receptor 4. J. Gerontol. Ser. A Boil. Sci. Med Sci. 2006, 61, 388-393. [CrossRef]

36. Stewart, L.K.; Flynn, M.G.; Campbell, W.W.; Craig, B.A.; Robinson, J.P.; McFarlin, B.K.; Timmerman, K.L.; Coen, P.M.; Felker, J.; Talbert, E. Influence of exercise training and age on CD14+ cell-surface expression of toll-like receptor 2 and 4. Brain Behav. Immun. 2005, 19, 389-397. [CrossRef]

37. Rodriguez-Miguelez, P.; Fernandez-Gonzalo, R.; Collado, P.S.; Almar, M.; Martínez-Flórez, S.; De Paz, J.A.; González-Gallego, J.; Cuevas, M.J. Whole-body vibration improves the anti-inflammatory status in elderly subjects through toll-like receptor 2 and 4 signaling pathways. Mech. Ageing Dev. 2015, 150, 12-19. [CrossRef]

38. Farinha, J.B.; Steckling, F.M.; Stefanello, S.T.; Cardoso, M.S.; Nunes, L.S.; Barcelos, R.P.; Duarte, T.; Kretzmann, N.A.; Mota, C.B.; Bresciani, G.; et al. Response of oxidative stress and inflammatory biomarkers to a 12-week aerobic exercise training in women with metabolic syndrome. Sports Med. Open 2015, 1, 1640. [CrossRef]

39. Pandey, K.B.; Rizvi, S.I. Markers of oxidative stress in erythrocytes and plasma during aging in humans. Oxidative Med. Cell. Longev. 2010, 3, 2-12. [CrossRef]

40. Fisher-Wellman, K.; Bloomer, R.J. Acute exercise and oxidative stress: A 30 year history. Dyn. Med. $2009,8,1$. [CrossRef]

41. Krause, M.; Rodrigues-Krause, J.; O’Hagan, C.; Medlow, P.; Davison, G.; Susta, D.; Boreham, C.; Newsholme, P.; O'Donnell, M.; Murphy, C.; et al. The effects of aerobic exercise training at two different intensities in obesity and type 2 diabetes: Implications for oxidative stress, low-grade inflammation and nitric oxide production. Eur. J. Appl. Physiol. 2014, 114, 251-260. [CrossRef]

42. Samjoo, I.A.; Safdar, A.; Hamadeh, M.J.; Raha, S.; Tarnopolsky, M.A. The effect of endurance exercise on both skeletal muscle and systemic oxidative stress in previously sedentary obese men. Nutr. Diabetes 2013, 3, e88. [CrossRef] [PubMed]

43. Campbell, P.T.; Gross, M.D.; Potter, J.D.; Schmitz, K.H.; Duggan, C.; McTiernan, A.; Ulrich, C.M. Effect of exercise on oxidative stress: A 12-month randomized, controlled trial. Med. Sci. Sports Exerc. 2010, 42, 1448-1453. [CrossRef] [PubMed]

44. Simar, D.; Malatesta, D.; Mas, E.; Delage, M.; Caillaud, C. Effect of an 8-weeks aerobic training program in elderly on oxidative stress and HSP72 expression in leukocytes during antioxidant supplementation. J. Nutr. Heal. Aging 2012, 16, 155-161. [CrossRef] [PubMed] 
45. Done, A.J.; Traustadóttir, T. Aerobic exercise increases resistance to oxidative stress in sedentary older middle-aged adults. A pilot study. AGE 2016, 38, 505-512. [CrossRef] [PubMed]

46. Krzanowski, M.; Krzanowska, K.; Gajda, M.; Dumnicka, P.; Dziewierz, A.; Woziwodzka, K.; Litwin, J.A.; Sułowicz, W. Pentraxin 3 as a new indicator of cardiovascular-related death in patients with advanced chronic kidney disease. Pol. Arch. Intern. Med. 2017, 127, 170-177. [PubMed]

47. Fernandez-Gonzalo, R.; De Paz, J.A.; Rodriguez-Miguelez, P.; Cuevas, M.J.; González-Gallego, J. TLR4mediated blunting of inflammatory responses to eccentric exercise in young women. Mediat. Inflamm. 2014, 2014, 1-11. [CrossRef]

48. Fernandez-Gonzalo, R.; De Paz, J.A.; Rodriguez-Miguelez, P.; Cuevas, M.J.; González-Gallego, J. Effects of eccentric exercise on toll-like receptor 4 signaling pathway in peripheral blood mononuclear cells. J. Appl. Physiol. 2012, 112, 2011-2018. [CrossRef]

(C) 2020 by the authors. Licensee MDPI, Basel, Switzerland. This article is an open access article distributed under the terms and conditions of the Creative Commons Attribution (CC BY) license (http://creativecommons.org/licenses/by/4.0/). 